\title{
Method of Flatness Pattern Recognition Based on Chaos Particle Swarm Algorithm Optimization Elman Network
}

\author{
Zhimin $\mathrm{Bi}^{\mathrm{a}}$, Yan Wang ${ }^{\mathrm{b}}$ \\ School of Control Science and Engineering, Jinan University, Jinan, 250022, China \\ a969543630@qq.com, b272508613@qq.com
}

\begin{abstract}
In order to obtain flatness pattern recognition method of high accuracy and simple operation .this paper presents a chaotic particle swarm initialization sequence, as well as put the chaotic sequence join into the thin iterative search process after meeting some conditions, Elman neural network shape pattern recognition method to improve the convergence speed and accuracy. In neural network modeling process, using chaotic particle swarm optimization algorithm global search for the best advantage of the ability to obtain the optimal network parameters optimized to improve accuracy of the flatness pattern recognition.
\end{abstract}

Keywords: Flatness pattern recognition; Elman neural network; Chaos particle swarm algorithm.

\section{Introduction}

Plate and strip production of which the national economy is an important basic raw material. Flatness is an important quality index of plate and strip, flatness pattern recognition as an important content of the flatness theory and plane degree is an vital part of control system, its main task and goal is to decompose the measured signal which is detected by flatness detecting device into a set of plate with transverse stress control agency for flatness controlling several basic flatness defect pattern, thus to provide evidence for drawing up a reasonable flatness control strategy [1]. In recent years, people put forward many new flatness pattern recognition methods, Peng Yan [2] presentes a flatness pattern recognition method based on the EBP network. Yin Guofang [3] proposed to the neural network pattern recognition such as simplex optimization method, improves the recognition accuracy, latness pattern recognition is proposed by Zhang Xiuling [4], such as GA - BP model, which solved the problem of neural network structure when width changes, and realizes the intelligent flatness recognition of flatness pattern recognition, but the identification error is bigger [5]. But, these methods rely on a large number of test data for training. To this end, the author uses the chaos particle swarm algorithm to optimize Elman neural network is used to identify the flatness and analysis.

\section{Elman network}

Elman type recurrent neural network is a dynamic recurrent neural network. Generally divided into four layers: an input layer, a hidden layer, state layer and output layer. The delay function of state layer is used to remember the hidden layer unit output data of the previous time, making the network has the a bility to adapt to the time-varying characteristics [6].

Elman network can be described as follows:

$$
\begin{aligned}
& y(k)=g\left(w^{3} x(k)\right) \\
& x(k)=f\left(w^{1} x_{c}(k)+w^{2}(u(k-1))\right) \\
& x_{c}(k)=x(k-1)
\end{aligned}
$$

The above formula (1) (2) (3): $y, x, u, x_{c}$ denote $m$ dimensional output vector, $n$-dimensional vector of the hidden layer, $r$-dimensional input vector and the $n$-dimensional state vector feedback; $W^{3}, W^{2}$, $W^{l}$ represent respectively the hidden layer to the output layer, the input layer to the hidden layer, layer to the status of the connection weights hidden layer. $g(\cdot)$ is the output neuron transfer function and a linear combination of the intermediate layer output, $f(\cdot)$ for the intermediate layer neuron transfer function, often with the $S$ function[7]. 


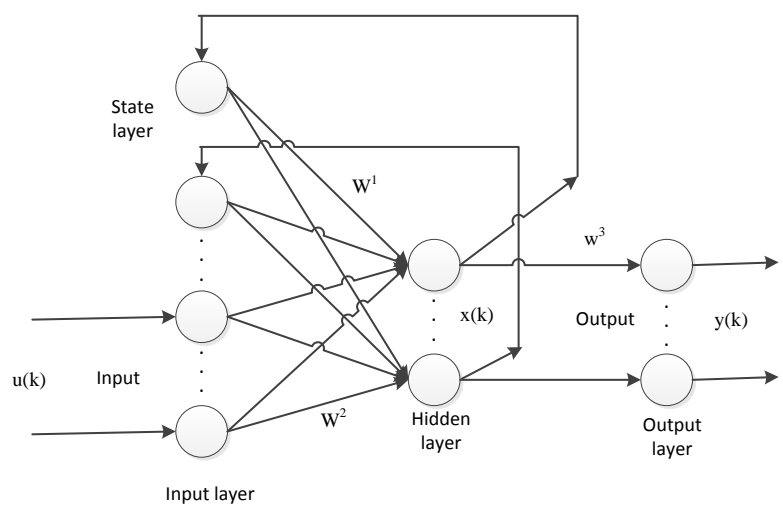

Fig.1 Elman neural network model

Elman network objective function by means of the function of error sum of squares:

$$
E(W)=\sum_{k=1}^{n}\left[y_{k}(w)-y(w)\right]^{2}
$$

In the above formula (4): $y(w)$ is the actual output vector.

\section{The CPSO algorithm optimize__ Elman network}

Particle swarm algorithm is a global search algorithm, each particle in the algorithm represents a potential solution to the problem. The characteristic of traversing, which chaotic search possess can avoid local extreme point, therefore, it usually requires a lot of iteration steps to get a better solution. The particle swarm algorithm is simple to operate, but easy to lead to local minima. Thus, we tried to combine chaos optimization algorithm with Particle swarm algorithm, in order to initialize the sequence by the Chaos particle swarm, besides, when certain conditions are met after the iterative search process, then fine chaotic sequence was added to give a chaotic particle swarm algorithm.

Logistic equation is a typical chaotic systems:

$$
\mathrm{z}_{n+1}=\mu \mathrm{z}_{n}\left(1-\mathrm{z}_{n}\right), n=0,1,2, \ldots
$$

In the formula: $\mu$ as a control parameter, $\mu=4$,system is completely in a state of chaos, Logistic mapping for full on $(0,1)$ interval mapping[8].

Particle swarm algorithm is updated by the velocity and position of the particle, so that the optimal solution is found in the solution space.In the particle swarm algorithm, we assume that the search space is $D$-dimensional,there are n particles.Particle in the space, the position of the $i$-th swarm in the group can be indicated with $X_{i}=\left[x_{i 1}, x_{i 2}, x_{i 3}, \ldots x_{i D}\right]$, and the speed of the $i$-th particle is expressed as $V_{i}$ $=\left[v_{i l}, v_{i 2}, v_{i 3}, \ldots v_{i D}\right]$. The best location of the $i$-th particle experienced is the $p_{i}=\left[p_{i l}, p_{i 2}, \ldots, p_{i D}\right]$, called individual extreme $P_{\text {best }}$, the best place that the entire particle swarm have searched so far is recorded as $p_{g}=\left[p_{g 1}, p_{g 2}, \ldots, p_{g D}\right]$,called global extreme $g_{\text {best }}[9]$.Each iteration, the velocity and position of the particle update formula is as follows:

$$
\begin{aligned}
& V^{k+1}{ }_{i d}=\mathrm{w} V^{k}{ }_{i d}+c_{1} r_{1}\left(P^{k}{ }_{i d}-X^{k}{ }_{i d}\right)+c_{2} r_{2}\left(P^{k}{ }_{g d}-X^{k}{ }_{i d}\right) \\
& X^{\mathrm{k}+1}{ }_{i d}=X^{k}{ }_{i d}+V^{k+1}{ }_{i d}
\end{aligned}
$$

$i=1,2, \ldots, n ; d=1,2, \ldots, D . w$ is a weighting factor ranging between $0.4 \sim 0.9 ; c_{1}, c_{2}$ are studying factors; $r_{1}$ and $r_{2}$ are uniformly distributed random number between $[0,1]$. Its limited to the maximum rate of the particles is $v_{\max }$, and the location of the particle within permissible range.

The process of Chaotic particle swarm optimization algorithm is as follows:

Step 1: Initialize Chaos particle position and velocity. Firstly, randomly generate a $D$-dimensional vector, use the (5) formula to give the $\mathrm{n}$ vectors $z_{i}$ and.Then it becomes a variable value interval corresponding to the carrier wave vector $x_{i, j}$, and randomly generated initial velocity, and its number is $n$.

Step 2: Individual extreme point of each particle is supposed to express its current position, and 
calculate the corresponding individual extreme; and selected the best as The global best $\mathrm{n}$ individuals from extreme value, and set the global extreme point as The current position of the particle.

Step 3: According to (6) (7) to update the particle's speed and position.

Step 4: If the updated particle fitness is better than individual extreme $P_{\text {best }}$, update the current individual extreme; if the updated particle fitness is better than global extremum $g_{\text {best }}$, update the current global minimum.

Step 5: If the number of iterations reach a certain value or meet the requirements, then stop the process of particle swarm optimization, the current global extreme points are used to the search of small perturbations to chaos, then conversely return Step 3.

Step 6: Define $k=1$, according to formula $x_{i, j}^{\prime}=p_{g j}+\operatorname{randn} x_{i, j}$ to do the second carrier, the new adaptation value and the global extreme value are compared and update global minimum if optimize. And randn $\mathrm{x}_{\mathrm{i}, \mathrm{j}}$ is a small section traversing chaotic variables, $p_{g}$ is the current global extreme points.

Step 7: Continue iterative search through the chaotic variable secondary carrier. The new adaptation value and the global extreme value are compared, if you update the global optimum extreme value, $k=k+1$. If the termination condition is satisfied then stop searching, output the optimal solution $p_{g}$, global extreme $g_{\text {best }}$; if not , return Step 6.

The optimized parameters initialization of the network, the gradient descent method is used to better tune the training, until meet the training accuracy.

\section{The Establishment of Flatness Recognition Model}

Typically,flatness defect pattern is more complex, difficult to precise mathematical description Its usual to see defining several simple flatness defects as a basic flatness model in the production, the complex strip shape is expressed as a combination of one or more basic mode.First, the measurement data processing, according to a discrete set of flatness flatness target $\sigma_{A i}$ and a set of measured stress signal $\sigma_{T i}(i=1,2,3, \ldots, m)$ to calculate stress flatness deviation, and all the data normalization normalized, and then decompositing the flatness measurement signal mode based on Legendre orthogonal polynomials[10].
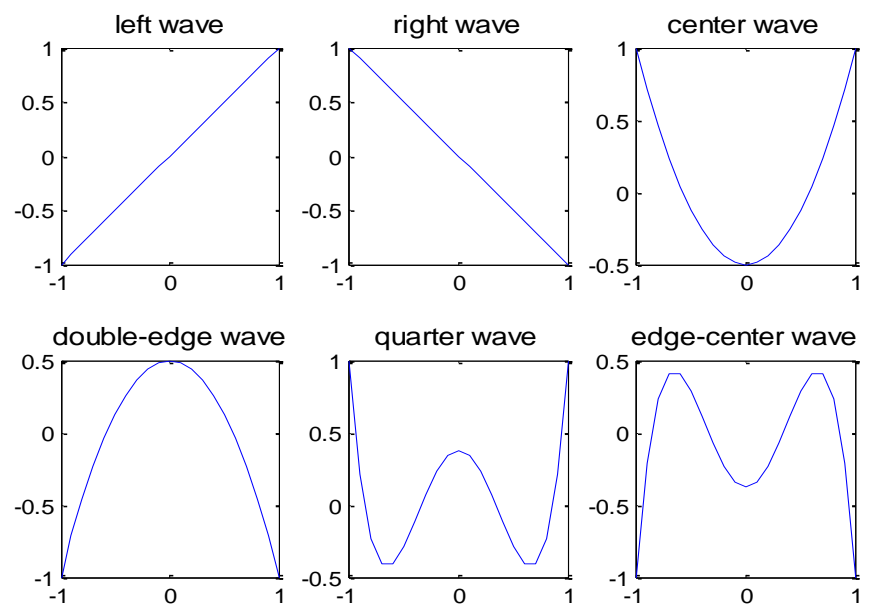

Fig.2 Plate-shaped basic pattern

The difference between the actual plate-like stress value $\sigma_{T i}$ and the standard stress value $\sigma_{A i}$ is taken as the plate-like deviation, and the maximum value $\Delta \sigma_{\max }$ of the plate-shaped deviation is selected. The result of the normalization of the plate-shaped deviation is $\sigma=\Delta \sigma_{i} / \Delta \sigma_{\max }$. After rolling flatness can use the following basic linear combination of the flatness model say:

$$
\sigma(x)=a_{1} P_{1}(x)+a_{2} P_{2}(x)+a_{3} P_{3}(x)
$$

In the above formula: $\sigma(x)$ is a longitudinal residual stress plate strip, $a_{1}, a_{2}, a_{3}$ is the characteristic parameters.Coefficients $\mathrm{a}_{1}, \mathrm{a}_{2}$ and $\mathrm{a}_{3}$ are the signs of different flatness defect pattern, such as the left represents the wave, the right wave, middle wave, bilateral waves, wave and quarter wave sides, the size of its numerical descriptes the proportion of such defects in the whole flatness defect. 
Use of unknown samples and six basic pattern between the Euclidean distance as the identification model of input, can be achieved under the condition of the plate width change recognition model input dimension is changeless, therefore, using Euclidean distance as the identification model of input.Euclidean distance between the normalized sample $Y$ and the $k$-th standard sample $Y_{k}: D_{K}=\left\|Y-Y_{K}\right\|=\sqrt{\sum_{i=1}^{m}\left(\sigma(i)-\sigma_{k}(i)\right)^{2}}(k=1,2, \ldots 6)$.Let the measured unknown pattern normalized $Y=(\sigma(1), \sigma(2), \ldots, \sigma(\mathrm{m})), \sigma(i)$ a stress value, $Y_{k}(\mathrm{k}=1,2,3,4,5,6)$ as the basic model of flatness standard samples. The $n$ standard samples are normalized to $Y_{k}=\left(\sigma_{k}(1), \sigma_{k}(2), \ldots, \sigma_{k}(m)\right), k=1,2, \ldots, n$; $m$ is the number of segments after discretization [11]. After Euclidean distance normalization: $D D_{k}=\frac{D_{K}}{\sum D_{k}}(k=1,2, \ldots 6) . D D_{k}$ is taken as the input of the recognition model, and the shape characteristic parameters $a_{1}, a_{2}$ and $a_{3}$ are taken as the output of the recognition model to establish the plate shape recognition model.

\section{Simulation and Analysis}

In order to verify the plate shape recognition capability of Chaotic particle swarm algorithm to optimize Elman network, using two kinds of methods to proceed pattern recognition of plate shape defect and compare the results, and using the Matlab Neural Network Toolbox to program simulation. Chaotic particle swarm algorithm to optimize the Elman network flatness pattern recognition method (referred to as CPSO-Elman), for comparison with the BP network Flatness Pattern Recognition (referred to as BP). It use the Euclidean distance $\left(D D_{K}\right)$ of identifying sample $Y$ with six standard samples as the input of the network, the plate-shape feature parameters $a_{1}, a_{2}, a_{3}$ as the output of the network.For the above two methods to compare the advantages and disadvantages under the same parameters, according to empirical formula and practice, selected the number of hidden layer neurons were 20, sample number select 100 groups, error precision is $10^{-5}$, learning rate is 0.01 , the number of training is 2000, the training step lengthis set to 10 .

The number of the Elman network layer neurons is 20. Its optimization algorithm parameters: $c_{1}=$ $1.49445, c_{2}=1.49445$; the population size is 50; the maximum evolution generation is 100; individual minimum and maximum value: popmax $=1$, popmin $=-1$; maximum and minimum speed value: $v_{\max }$ $=1, v_{\min }=-1$.

As shown in figure 3 (a) (b), the number of the identified training precision needed is 129 and 52 after the BP network and the optimized Elman network. Through the comparison proves that the optimized Elman network training performance is better than that of the BP network, the training time is shorter.
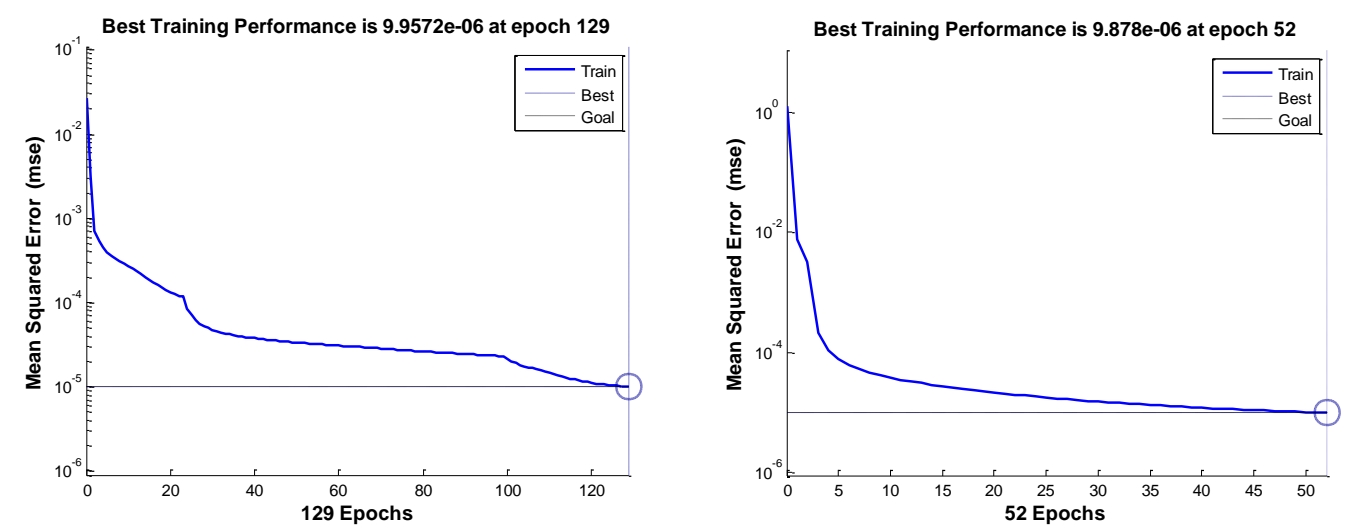

Fig.3 (a) BP performance training Fig.3 (b) CPSO-Elman performance training

Two methods of training samples for the previous six standard flatness defect pattern, its network recognition results are shown in table 1 below. 
Table 1 Flatness recognition result

\begin{tabular}{|c|c|c|c|c|c|c|c|c|c|}
\hline \multirow{2}{*}{ Test sample } & \multicolumn{3}{|c|}{ Standard output } & \multicolumn{3}{|c|}{$\mathrm{BP}$} & \multicolumn{3}{|c|}{ CPSO-Elman } \\
\hline & $a_{1}$ & $a_{2}$ & $a_{3}$ & $a_{1}$ & $a_{2}$ & $a_{3}$ & $a_{1}$ & $a_{2}$ & $a_{3}$ \\
\hline$Y=0.7 Y_{2}+0.3 Y_{6}$ & -0.7 & 0 & -0.3 & -0.7431 & 0.0214 & -0.2698 & -0.7011 & 0.007 & -0.3012 \\
\hline$Y=0.8 Y_{2}+0.2 Y_{4}$ & 0.8 & -0.2 & 0 & 0.8409 & -0.2273 & 0.0301 & 0.8007 & -0.2010 & 0.0008 \\
\hline$Y=0.5 Y_{1}+0.5 Y_{5}$ & 0.5 & 0 & 0.5 & 0.4731 & 0.0197 & 0.5186 & 0.5006 & 0.0011 & 0.4989 \\
\hline$Y=0.1 Y_{1}+0.4 Y_{4}+0.5 Y_{5}$ & 0.1 & -0.4 & 0.5 & 0.0749 & -0.4367 & 05201 & 0.1009 & -0.3990 & 0.4991 \\
\hline
\end{tabular}

As can be seen from Table 1, we can identify the samples by using the two methods mentioned above and predict the size of the plate-shaped characteristic parameters, but the precision by using CPSO-Elman to identify the plate-shaped defect types is significantly higher than that of the BP network. In order to more intuitive verify CPSO-Elman effectiveness and accuracy of the identification method, get a set of normalized after the measured data of $\sigma=[0-0.2720-0.3805-0.3700$

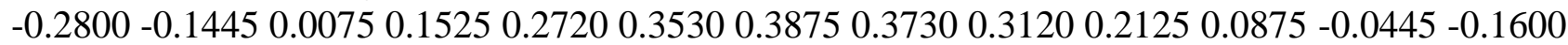
$-0.2300-0.2205-0.09200 .2000]$. The above two methods are used to identify the plate shape recognition results in a curve form, as shown in Figure 4 .The curve in Fig. 4 shows that CPSO-Elman is more accurate than the BP network in identifying the defect types.

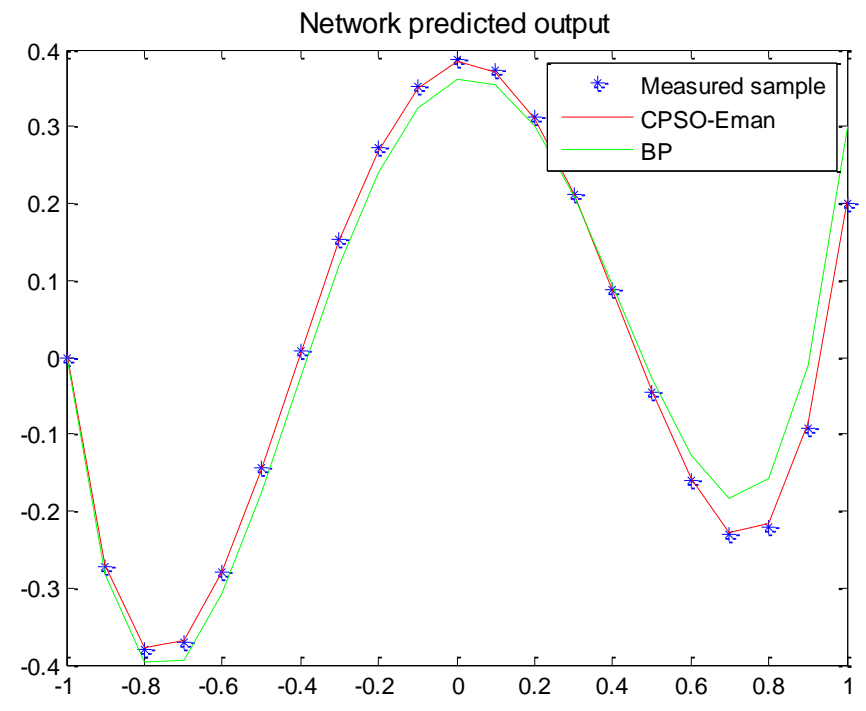

Fig. 4 Network predicted output

\section{Summary}

This paper presents a method that chaotic particle swarm optimize the Elman network flatness pattern recognition: It produce populations by its ergodic and random of chaos, its value is evenly distributed in the solution space and can produce a better solution at the beginning iterations, speed up the search speed and improve the convergence rate, we add the chaotic sequence in careful search after iterative optimization meet certain conditions and avoid the algorithm trapped in local optimum and improve the accuracy of the algorithm. The flatness defect recognition by using chaotic particle swarm optimization algorithm has the high precision ,significantly better than BP network flatness and achieve the desired effect.

\section{References}

[1] ZHAO Xiao-yan, ZHANG Zhao-hui, LAN Jin-hui. Flatness pattern recognition based on a binary tree hierarchical BP model. Journal of University of Science and Technology Beijing. Vol. 31 (2009) No. 2, p. 261-271.

[2] PENG Yan: Theoretical studies and engineering application of shape preset control for HC cold mill based on strip element method (Doctor of Engnieering, Yanshan University, China 2000). 
[3] YIN Guofang, WANG Yiqun, SUN Xuguang. Research on shape signal pattern recognition based on neural network. China Mechanical Engineering. Vol. 15 (2004) No. 24, p. 15-18.

[4] ZHANG Xiuling, LIU Hongmin. GA-BP model of flatness pattern recognition and improved least-squares method. Iron \& Steel. Vol. 10 (2003) No. 38, p. 29-34.

[5] LIU Jian, WANG Yiqun, SUN Fu, NING Shurong. Fuzzy Pattern Recognition Method of Flatness Based on Particle Swarm Theory. Chinese Journal of Mechanical Engineering. Vol. 44 (2008) No. 1, p. 173-178.

[6] Xie Qingguo, Wan Shuyun, Zhaojin. A systemic design for Elman recurrent network. J. Huazhong Univ. of Sci \&Tech.Vol.30 (2002) No. 3, p. 22-24.

[7] HUANG Yu-chun, TIAN Jian-ping, YANG Hai-li, HU Yong, ZHANG Liang-dong. Thermal Error Modeling for Machine Tool Based on Genetic Algorithm Optimization Elman Neural Network. Modular Machine Tool \& Automatic Manufacturing Technique. Vol. 4 (2015) No. 4, p. 74-77.

[8] WANG Xingyuan, LUO Chao. Dynamic Analysis of the Coupled Logistic Map. Journal of Software. Vol. 17 (2006) No. 4, p. 729-739.

[9] ZHOU Xiuxiu, CHANG Anding, GUO Jianqing, WANG Jiujie. Application of chaos particle swarm optimization algorithms to estimation of aquifer parameters. Journal of Water Resources \& Water Engineering. Vol. 24 (2013) No. 1, p. 96-99.

[10]ZHANG Xiuling, ZHAO Wenbao, ZHANG Shaoyu, XU Teng. Method of flatness pattern recognition based on improved T-S cloud inference network. Journal of Central South University: Science and Technology. Vol. 44 (2013) No. 2, p. 580-586.

[11]XIE Yangpeng, YANG Lushan. Study on flatness pattern recognition based on intelligent optimal radial basis function neural network. Juournal of Zhengzhou University of light industry: Natural Science. Vol. 27 (2012) No. 3, p. 89-92. 